Chirurg 2022 $93: 200$

https://doi.org/10.1007/s00104-022-01579-x

Angenommen: 7. Januar 2022

Online publiziert: 18. Januar 2022

(c) The Author(s), under exclusive licence to Springer Medizin Verlag GmbH, ein Teil von Springer Nature 2022

\section{Klassifikation der intraoperativen Schwierigkeit offener Leberteilresektionen}

\author{
Oliver Rohland · Michael Ardelt · Utz Settmacher \\ Klinik für Allgemein-, Viszeral- und Gefäßchirurgie, Universitätsklinikum Jena, Jena, Deutschland
}

Originalpublikation

Pothet C, Drumez , Joosten A et al (2021) Predicting Intraoperative Difficulty of Open Liver Resections. Ann Surg 202(1). https://doi. org/10.1097/SLA.00000000000005133.

Hintergrund. Bisherige Versuche die offene Leberteilresektion (LR) nach Schwierigkeitsgrad zu klassifizieren, waren nur auf das postoperative Outcome fokussiert und haben bis auf die vorgegebene anatomische Lokalisation andere anatomische und technische Faktoren nicht betrachtet. Das Ziel dieser Arbeit war es, ein Prognosemodel zu generieren, welches vorhersagen soll, wie anspruchsvoll eine LR sein wird.

Methodik. Anhand einer prospektiven Datenbank von insgesamt sechs französischen Zentren wurden die Qualität und das Outcome von LR ausgewertet. Von den Autoren wurden vier intraoperative Variablen ausgewählt, welche die Schwierigkeiten widerspiegeln sollen: die Operationszeit, die Gewebsdissektionszeit, der Blutverlust und ob ein Pringle-Manöver durchgeführt wurde. Es wurde eine hierarchische Clusteranalyse (HAC) durchgeführt, um homogene Gruppen basierend auf den vier Variablen zu identifizieren. Um die Gruppe mit der höchsten Schwierigkeit zu finden, wurden die vorher definierten

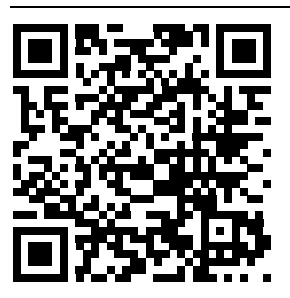

QR-Code scannen \& Beitrag online lesen technischen und anatomischen Faktoren ausgewählt, um damit eine multivariable logistische Regression (DIFF-scOR) durchzuführen. Hierbei wurde das Model nach Diskrimination und Kalibration bewertet und fehlende Daten mittels Imputation vervollständigt.

Ergebnisse. Die HAC hat zwei Cluster mit besonders hoher operativer Schwierigkeit identifiziert. In der Gruppe der „schwierigen LR" (20,8\% der Eingriffe) war die Operationszeit länger (401 min vs. $243 \mathrm{~min}$ ), die Gewebsdissektionszeit länger (150 min vs. $63 \mathrm{~min})$, der Blutverlust größer ( $900 \mathrm{ml}$ vs. $400 \mathrm{ml}$ ) und das Pringle-Maneuver wurde öfter angewendet (3 vs. 1) als in der Gruppe der "Standard-LR". Die ausschlaggebenden Faktoren in der Analyse dafür waren: das Körpergewicht, die Anzahl und Größe der Tumorknoten, das Anlegen einer Gallenwegsdrainage, Durchführung einer anatomischen oder kombinierten LR, Verlauf der Gewebstranssektionsebene zwischen den Segmenten II und IV oder IV und VIII oder VII und VIII, nichtanatomische Resektionen der Segmente II oder VII oder VIII, Resektion der Vena cava inferior, Anlage einer biliodigestiven Anastomose und eine hohe Anzahl der resezierten Lebersegmente. Die c-Statistik des DIFFscOR lag bei 0,822. Im Gegensatz dazu war die Vorhersage der 90-Tage-Mortalität und Morbidität des DIFF-scOR eher schlecht (cStatistik: 0,616 und 0,634).

\section{Kommentar}

Der DIFF-scOR sagt die Schwierigkeit einer offenen Leberteilresektion akkurat vorher. Zukünftig soll diese auch für andere Pro- zeduren wie z. B. Lebertransplantationen Anwendung finden.

\section{Korrespondenzadresse}

PD Dr. med. habil. Dr. med. univ. Michael Ardelt

Klinik für Allgemein-, Viszeral- und Gefäßchirurgie, Universitätsklinikum Jena Am Klinikum 1, 07747 Jena, Deutschland michael.ardelt@med.uni-jena.de

Interessenkonflikt. O. Rohland, M. Ardelt und U. Settmacher geben an, dass kein Interessenkonflikt besteht. 